\title{
Transferrin uptake may occur through detergent-resistant membrane domains at the cytopharynx of Trypanosoma cruzi epimastigote forms
}

\author{
José R Corrêa, Georgia C Atella*, Camila Vargas*, Maurilio J Soares**/+ \\ Laboratório de Ultra-estrutura Celular, Instituto Oswaldo Cruz-Fiocruz, Rio de Janeiro, RJ, Brasil *Instituto de Bioquímica Médica, \\ CCS, Universidade Federal do Rio de Janeiro, Rio de Janeiro, RJ, Brasil **Instituto de Biologia Molecular do Paraná-Fiocruz, Rua \\ Prof. Algacyr Munhoz Maeder 3775, Cidade Industrial, 81350-010 Curitiba, PR, Brasil
}

Uptake of transferrin by epimastigote forms of the protozoan Trypanosoma cruzi occurs mainly through a cytostomel cytopharynx, via uncoated endocytic vesicles that bud off from the bottom of the cytopharynx. We have here examined whether detergent-resistant membrane (DRM) domains might be involved in this process. Purified whole cell membrane fractions were assayed for cholesterol levels and used in dot blot analyses. Detergent-resistant membrane markers (cholera B toxin and anti-flotillin-1 antibody) presented positive reaction by dot blots in cholesterolrich/ protein-poor membrane sub-fractions. The positive dot blot fraction was submitted to lipid composition analysis, showing composition similar to that of raft fractions described for other eukaryotic cells. Immunofluorescence assays allowed the localization of punctual positive signal for flotillin-1, matching the precise cytostomel cytopharynx location. These data were confirmed by immunofluorescence assays with the co-localization of flotillin-1 and the transferrin uptake site. Our data suggest that DRM domains occur and are integrated at the cytostome/ cytopharynx of $\mathrm{T}$. cruzi epimastigotes, being the main route for transferrin uptake.

Key words: cytopharynx - detergent-resistant membrane - endocytosis - Trypanosoma cruzi - ultrastructure

The lipid raft model proposes that cholesterol and sphingolipids of the plasma membrane outer leaflet are not randomly distributed, but rather clustered into micro-domains that float in the lipid bilayer, characterizing detergent-resistant membrane (DRM) domains in eukaryotic cells (Rietveld \& Simons 1998, Handcock 2006). Transmembrane proteins (receptors, adhesion molecules, and enzymes) in the lipid bilayer would either reside in, and thus become sorted into transport vesicles, or be excluded from the rafts, depending on partitioning imparted by their physical properties (Harder et al. 1998, Simons \& Vaz 2004).

Lipid rafts play a role in a wide range of important biological processes, including signal transduction pathways, apoptosis, cell adhesion and migration, organization of the cytoskeleton and protein sorting during both exocytosis and endocytosis (Simons \& Toomre 2000, Harris \& Siu 2002).

Flotillins are cholesterol-binding proteins that associate with a subset of rafts named caveolae, and are frequently used as raft markers. The flotillin protein family consists of the small $(45 \mathrm{kDa})$ proteins flotillin-1 and flotillin-2/ ESA (a close homologue of the epidermal

Financial support: CNPq, Faperj, Papes IV-Fiocruz, International Foundation for Sciences (IFS).

+Corresponding author: maurilio@tecpar.br

Received 6 July 2007

Accepted 6 October 2007 surface antigen). These proteins induce caveolae formation, bind cholesterol, and interact with signaling molecules (Anderson 1998, Kiss et al. 2004). Lipid rafts can exist independently of caveolae, but they must exist prior to formation of caveolae for proper insertion of flotillin into membranes (Graf et al. 1999, Ikonen 2001).

Trypanosomatid protozoa have a particular cell body array that allows uptake of nutrients only at a specific membrane site: the flagellar pocket (Morgan et al. 2001). Trypanosoma cruzi, the causative agent of Chagas disease in Latin America, appears as an attractive model for studies to elucidate the occurrence of lipid rafts and their probable participation in endocytic processes, as T. cruzi epimastigotes have a second specialized endocytic site, the cytostome/ cytopharynx. This is a plasma membrane invagination located at the anterior cell end, which penetrates deeply into the cytoplasm towards the nucleus (Milder \& Deane 1969). Uncoated vesicles have been observed budding off from the flagellar pocket membrane (Soares et al. 1992) and the bottom of the cytopharynx (Figueiredo \& Soares 2000), thus demonstrating the occurrence of endocytosis by uncoated vesicles in T. cruzi epimastigotes. Protein uptake through receptor-mediated endocytosis also occurs (Soares \& De Souza 1991, Figueiredo \& Soares 2000), with ingestion of transferrin mainly through the cytostome (PortoCarreiro et al. 2000). The molecules involved in vesicle budding remain poorly known, but recent studies indicate a functional role for lipid rafts in trypanosomatids (Denny \& Smith 2004). Here we identify in T. cruzi epimastigotes some lipid rafts landmarks and present evidences for the presence of a DRM site involved in endocytosis at the cytostome/ cytopharynx. 


\section{MATERIALS AND METHODS}

Parasites - Epimastigote forms of T. cruzi, strain Y, were kept axenically at $28^{\circ} \mathrm{C}$ by weekly transfers in liver infusion tryptose medium (Camargo 1964) supplemented with $10 \%$ heat inactivated fetal calf serum (CultiLab, São Paulo, Brazil). Five-day-old epimastigotes, at the mid$\log$ phase of growth, were used for the experiments.

DRM purification - The parasites were washed in phosphate buffered saline (PBS, pH 7.2) and TNE buffer (25 mM Tris- $\mathrm{HCl} \mathrm{pH} 7.4,150 \mathrm{mM} \mathrm{NaCl}, 5 \mathrm{mM}$ EDTA, $0.2 \mathrm{mM}$ PMSF, $1 \mu \mathrm{g} / \mathrm{ml}$ leupeptin, $1 \mu \mathrm{g} / \mathrm{ml}$ pepstatin, 1 $\mu \mathrm{g} / \mathrm{ml}$ aprotinin, $1 \mathrm{mM} \mathrm{Na}_{3} \mathrm{VO}_{4}$, and $1 \mathrm{mM} \mathrm{NaF}$ ).

Aliquots of $5 \times 10^{9}$ cells in TNE buffer were carefully disrupted on ice with a Branson Sonifier Cell disruptor B15 (Sonifier Cell Disruptors, Danbury, CT, USA) using a standard probe (13 $\mathrm{mm}$ radiating diameter) operating at $15 \%$ of total amplitude, with 5 cycles of $30 \mathrm{~s}$ and with $1 \mathrm{~s}$ rest between cycles, yielding a total homogenate. This homogenate was centrifuged at $20,000 \mathrm{~g}$ and the supernatant was incubated for $20 \mathrm{~min}$ at $4^{\circ} \mathrm{C}$ in TNE buffer containing $1 \%$ Triton X-100. After incubation, the sample was mixed $1: 1$ with $80 \%$ sucrose $(\mathrm{w} / \mathrm{v})$ in TNE buffer and transferred to a Beckman SW40 centrifuge tube (Beckman Coulter Inc, Fullerton, CA, USA). This mixture was first overlaid with $35 \%$ sucrose, followed by 5\% sucrose, and centrifuged at $100,000 \mathrm{~g}$ for $16 \mathrm{~h}$ at $4^{\circ} \mathrm{C}$ (Chung et al. 2005). Twelve 1-ml fractions could be sequentially collected from the top. Raft cholesterol was measured with a sensitive cholesterol oxidase-based fluorometric assay (Amplex Red cholesterol kit) purchased from Molecular Probes (Eugene, OR, USA). Cholesterol content of purified lipid rafts was normalized to total protein fraction.

Cholera B toxin and anti-flotillin-1 dot blotting About $0.1 \mathrm{ml}$ from each gradient fraction was diluted to $1 \mathrm{ml}$ with PBS and adsorbed onto a nitrocellulose filter using a dot-blot apparatus (Schleicher \& Schuell, Keene, $\mathrm{NH}$, USA). Membranes were blocked with 5\% non-fat dry milk and probed with a Horse Radish PeroxidaseCholera B Toxin (HRP-CtxB) conjugate (Invitrogen, Carlsbad, CA, USA) or else with anti-flotillin-1 antibody (Sigma Chemical Co, St Louis, MO, USA) for $1 \mathrm{~h}$ at room temperature. Blots were developed using a chemiluminescence assay (Pierce, Rockford, IL, USA).

Lipid analysis - Cholera B toxin positive fractions (4 to 6) were pooled and lipids were extracted (Bligh \& Dyer 1959) for $2 \mathrm{~h}$ in a stoppered tube containing $5 \mathrm{ml}$ of a chloroform-methanol-water solution $(2: 1: 0.8, \mathrm{v} / \mathrm{v})$, with intermittent agitation. After centrifugation, the supernatant was collected and the pellet subjected to a second lipid extraction for $1 \mathrm{~h}$. The amount of total lipids was determined gravimetrically. Extracted lipids were analyzed by one-dimensional high performance thinlayer chromatography (HPTLC) for neutral lipids (Vogel et al. 1962), or by two-dimensional thin-layer chromatography (TLC) for phospholipids (Yavin \& Zutra 1977). Sphingolipids were identified by mild alkaline hydrolysis of the total lipid extract for $1 \mathrm{~h}$ at $80^{\circ} \mathrm{C}$ in methanolic 2.5 M KOH. Neutral lipids were visualized by charring $\left(10 \mathrm{~min}\right.$ at $\left.110^{\circ} \mathrm{C}\right)$ after submersion of the plates for $10 \mathrm{~s}$ in $3 \% \mathrm{CuSO}_{4}$ in $8 \% \mathrm{H}_{3} \mathrm{PO}_{4}$. Lipids were globally quantified from HPTLC plates by densitometric scanning. Each lipid spot was identified by comparing to lipid standards (Sigma) run in parallel. A lipid mass curve was made to convert the densitometric values into $\mu \mathrm{g}$ of each lipid on the HPTLC plate.

Uptake of transferrin-gold complexes - Colloidal gold particles (15 nm in diameter) were prepared as described (Slot \& Geuze 1985). Human holo-transferrin (Sigma) was coupled to gold particles as described elsewhere (Bendayan et al. 1987). T. cruzi epimastigotes were collected by centrifugation at $1,500 \mathrm{~g}$, washed twice in PBS to remove the culture medium, and incubated for $30 \mathrm{~min}$ at $28^{\circ} \mathrm{C}$ with gold-labeled protein solutions diluted 1:1 in PBS. The cells were then processed for transmission electron microscopy.

Transmission electron microscopy - After the endocytosis assays, the parasites were rinsed with PBS, fixed for $1 \mathrm{~h}$ at room temperature with $2.5 \%$ glutaraldehyde in $0.1 \mathrm{M}$ phosphate buffer ( $\mathrm{pH} 7.2$ ), rinsed in phosphate buffer, and then post-fixed for 30 minutes with $1 \%$ osmium tetroxide/ $0.8 \%$ potassium ferricyanide/ $5 \mathrm{mM}$ $\mathrm{CaCl}_{2}$ in $0.1 \mathrm{M}$ cacodylate buffer ( $\mathrm{pH}$ 7.2). Thereafter, the parasites were rinsed in cacodylate buffer, dehydrated in a graded acetone series, and embedded in Poly/Bed 812 resin (PolySciences, Warrington, PA, USA). Ultrathin sections were stained with uranyl acetate and lead citrate and observed in a Zeiss (Oberkochen, Germany) EM10C transmission electron microscope.

Immunofluorescence assays - For the double fluorescence assay, epimastigotes were harvested, washed three times with PBS and incubated for $15 \mathrm{~min}$ at $28^{\circ} \mathrm{C}$ with $1 \mathrm{mg} / \mathrm{ml}$ TRITC (Tetramethyl Rhodamine Iso-Thiocyanate)-labeled transferrin (Sigma). The cells were then adhered at $4^{\circ} \mathrm{C}$ to $0.1 \%$ poly-L-lysine-coated slides and fixed for $20 \mathrm{~min}$ on ice with methanol at minus $20^{\circ} \mathrm{C}$. Thereafter, the slides were incubated for $10 \mathrm{~min}$ at room temperature with permeabilizing buffer [PBS with $0.2 \%$ $(\mathrm{w} / \mathrm{v})$ saponin, $1 \%(\mathrm{w} / \mathrm{v})$ bovine serum albumin, $5 \%(\mathrm{v} / \mathrm{v})$ fetal calf serum], blocked for $1 \mathrm{~h}$ at room temperature with blocking buffer $[4 \%(\mathrm{w} / \mathrm{v})$ bovine serum albumin in PBS], and then incubated for $1 \mathrm{~h}$ at room temperature with primary antibody against flotillin-1 diluted at 1:200 in blocking buffer.

The slides were washed with $1 \%$ bovine serum albumin in PBS, prior to incubation with a secondary AlexaFluor-488 (Invitrogen) antibody. The cells were then stained with $0.5 \mu \mathrm{g} / \mathrm{ml}$ DAPI and examined by confocal laser scanning microscopy.

\section{RESULTS}

Detection of lipid raft markers in detergent-insoluble membrane fractions - DRM extracts prepared with Triton X-100 were first used to analyze the distribution of cholesterol in sucrose density gradients after equilibrium centrifugation, as compared to the protein amounts. The DRM fractions were identified in the sucrose gradients as white-tan cloudy layers of material just above the middle of the gradient tub. Fractions 4 to 
6 , but mainly fraction 5 , showed enrichment in cholesterol with a low content of proteins, as described for lipid rafts in mammal cells (Fig. 1A).

Lipid raft markers (cholera B toxin reaction and flotillin1) were detected in the gradient fractions by dot-blot analysis. The flotillin-1 positive fractions matched with the cholera $\mathrm{B}$ toxin positive fractions, and both markers matched with the cholesterol-rich factions 4 to 6 (Fig. 1B).

Lipid analysis was undertaken to quantify the distribution of lipid classes in the DRM fractions 4 to 6 . As expected for lipid raft sub-domains, cholesteryl ester comprised about $45 \%$ of the total lipids, while ergosterol and triacylglycerol were the major neutral lipids (Fig. 2A). Furthermore, analysis of the polar lipid composition in these fractions showed that sphingolipids were highly enriched, whereas phosphatidylethanolamine and phosphatidylcholine were found in minor amounts (Fig. 2B).

Ultrastructure of budding endocytic vesicles - Morphological analysis by transmission electron microscopy of parasites incubated with gold-labeled transferrin showed the marker located along and at the bottom of the cytopharynx (Fig. 3A). The cytopharynx is a long tube that extends toward the posterior region of the cell, frequently ending past the nucleus, close to the reservosomes (Fig. 3B).

\section{A}

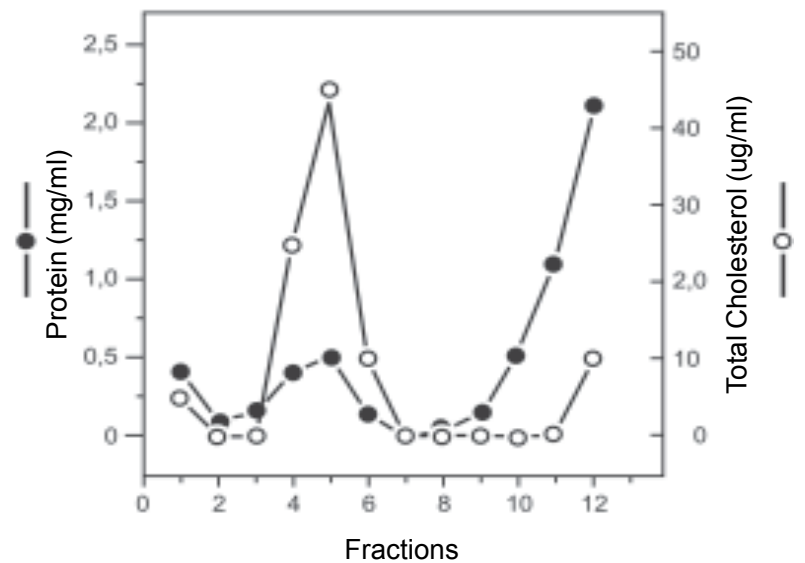

B

Flotillin

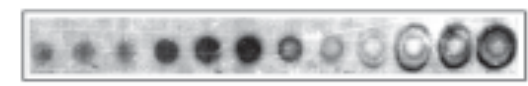

Cholera

toxin

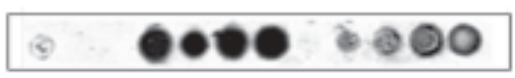

Gold particles were located bound to uncoated vesicles budding off from the bottom of the cytopharynx (Fig. 3A). These data indicated that a DRM site could be located at the cytostome/ cytopharynx, related to endocytosis of proteins.

Flotillin-1 detection and co-localization with the transferrin uptake site by immunofluorescence microscopy - Immunolabeled parasites showed positive, punctual flotillin-1 fluorescence signals in the vicinity of the

A $\%$

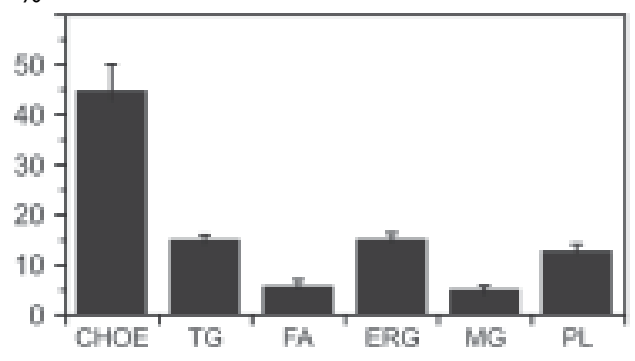

B $\%$

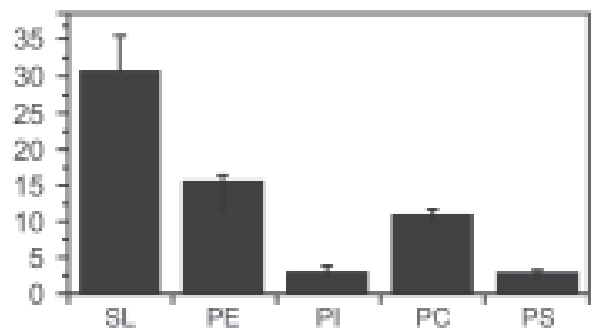

Fig. 2: lipid analysis of detergent-resistant membrane domains in Trypanosoma cruzi epimastigotes. Total lipids from fractions 4-6 were determined gravimetrically and the extracted lipids were then analyzed. Data are expressed as lipid component percentage. Results are the mean \pm standard deviation of three experiments. A: analysis by one-dimensional high performance thin-layer chromatography (HPTLC) for neutral lipids. CHOE: cholesterol ester; ERG: ergosterol; FA: free fatty acids; MG: monoacylglycerol; PL: phospholipids; TG: triacylglycerol. B: analysis by two-dimensional thin-layer chromatography (TLC) for phospholipids. PC: phosphatidylcholine; PE: phosphatidylethanolamine; PI: phosphatidy-linositol; PS: phosphatidylserine; SL: sphingolipids.
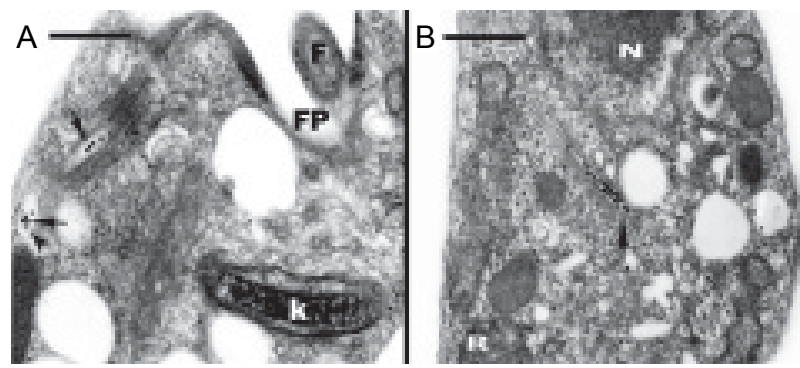

Fig. 3: ultrastructure of the uptake of gold-labeled transferrin by Trypanosoma cruzi epimastigotes at $28^{\circ} \mathrm{C}$. A: gold label (arrows) is found inside the cytopharynx and associated to an uncoated vesicle formed at its bottom (arrowhead); B: gold label is located along the cytopharynx (arrow) at the posterior region of the cell, past the nucleus $(\mathrm{N})$ and close to the reservosomes (R). F: flagellum; FP: flagellar pocket; K: kinetoplast. $\operatorname{Bar}=0.2 \mu \mathrm{m}$.
Fig. 1: total cholesterol/ proteins concentration and detection of raft markers in Trypanosoma cruzi epimastigotes. A: in detergent-resistant membrane fractions, cholesterol was enriched in sub-fractions 4 to 6 , which present a poor protein concentration; B: presence of raft markers flotillin-1 and GM1 was determined in each sub-fraction by dot blotting. Both markers were also enriched in fractions 4 to 6 , as expected for lipid raft domains. 
kinetoplast (stained blue with DAPI), at the posterior part of the cell (Fig. 4A-D). This region corresponds precisely to the cytostome opening in epimastigotes. Strong labeling was also located along and at the bottom (Fig. 4A-D) of the cytopharynx.

Association of transferrin uptake regions and flotillin-1 positive sites was visualized by co-localization of flotillin-1 fluorescent signals (green) and TRITC-
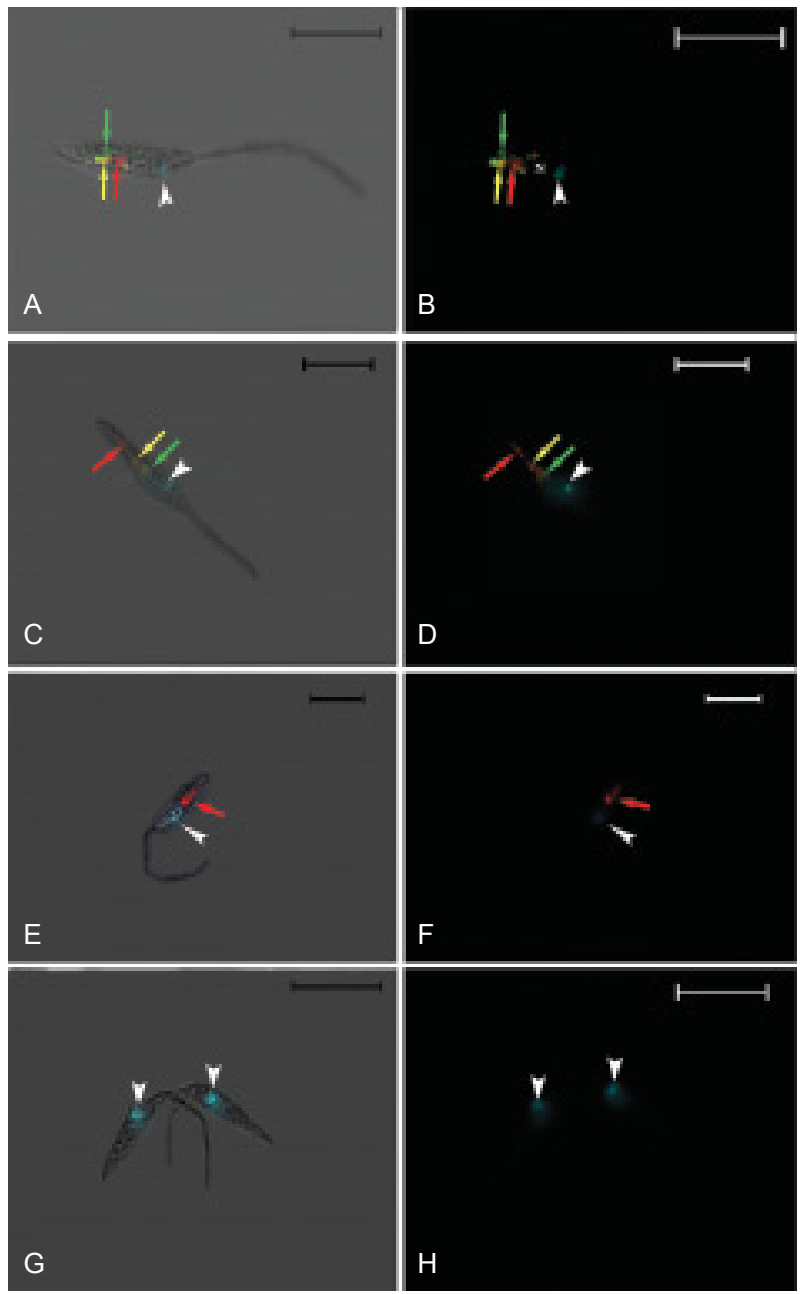

Fig. 4: immunofluorescence co-localization of TRITC-labeled transferrin and flotillin-1 in Trypanosoma cruzi epimastigotes. A, C: combined phase contrast image (cell body) and the confocal fluorescence images shown in B and D. The kinetoplast (arrowhead) is stained blue with DAPI. Colocalization of flotillin-1 (green arrow) and TRITC-labeled transferrin (red arrow) signals resulted in yellow color labeling (yellow arrow); B, D: confocal fluorescence images. The green arrow indicates the flotillin-1 location (green spots) at the middle and bottom portions of the cytopharynx. The red arrow shows TRITC-labeled transferrin (red spots) in the cytopharynx at the posterior end of the cell. The yellow arrow shows the merging of the two fluorescence signals (yellow spots) at the cytopharynx bottom. The kinetoplast/nuclear region (arrowhead) stained blue with DAPI; E - H: as negative controls, parasites were either first incubated with TRITC-labeled transferrin and then with the secondary antibody (E, F), or else they were incubated with the secondary antibody alone $(\mathrm{G}, \mathrm{H})$. The red arrow shows transferrin inside the cell; arrowheads show the kinetoplast/nuclear region. $\mathrm{Bar}=10 \mu \mathrm{m}$. labeled transferrin (red). Merged fluorescence of both markers at the cytostome bottom resulted in yellow color (Fig. 4A-D). Control parasites incubated with TRITClabeled transferrin and AlexaFluor-488 secondary antibody only (omission of the flotillin-1 antibody incubation step), showed no green or yellow fluorescence (Fig. $4 \mathrm{E}, \mathrm{F})$. Cells incubated with the secondary antibody alone showed no fluorescence signal, except for DAPI-stained nuclei and kinetoplasts (Fig. 4G, H).

\section{DISCUSSION}

Incubation of T. cruzi epimastigotes with gold-labeled proteins at $4^{\circ} \mathrm{C}$ demonstrated the occurrence of receptor-mediated endocytosis of transferrin through uncoated vesicles at the cytostome/ cytopharynx (Soares \& De Souza 1991, Figueiredo \& Soares 2000, PortoCarreiro et al. 2000). Formation of such uncoated vesicles, associated to the detection of typical lipid raft markers in epimastigotes, suggests that endocytosis of nutrients through the cytostome occurs via DRM domains and indicate that the cytostome/ cytopharynx is a distinct membrane sub-domain that may account for different mechanisms of recognition and receptor-mediated uptake of macromolecules.

The cytostome/ cytopharynx of T. cruzi is a plasma membrane invagination that penetrates deeply into the cytoplasm towards the nucleus (Milder \& Deane 1969). Fluid-phase pinocytosis of peroxidase and receptormediated endocytosis of transferrin occur through this structure, resulting in accumulation of ingested proteins in organelles called reservosomes (Soares \& De Souza 1988, Soares et al. 1992, Porto-Carreiro et al. 2000). Our biochemical analysis of a DRM fraction allowed the detection of flotillin-1 and a singular T. cruzi glycolipid that positively reacts with cholera B toxin, both labels considered as lipid raft markers (Nguyen \& Hildreth 2000, Seveau et al. 2004). Furthermore, we have also obtained immunofluorescence co-localization for flotillin-1 and TRITC-labeled transferrin in a region corresponding to the cytostome/ cytopharynx. Although no flotillin-1 gene has been found in the T. cruzi genome database (El-Sayed et al. 2005), the punctual positive reaction for flotillin-1 at the cytostome/ cytopharynx suggests that a similar protein might be present at this site, although the possibility of cross-reactivity with a non-related protein can not be excluded. Recent data from Hela and MEF cells have shown that coassembly of flotillin-1 and flotillin-2 into micro-domains induced membrane curvature, formation of plasma-membrane invaginations morphologically similar to caveolae, and the accumulation of intracellular vesicles. These microdomains are distinct from caveolin1-positive caveolae, are dynamic, and bud into the cell. It has been proposed that flotillin proteins are defining structural components of the machinery that mediates a clathrinindependent endocytic pathway (Frick et al. 2007).

Our morphological and biochemical data indicate that receptor-mediated endocytosis of transferrin by $T$. cruzi epimastigotes occurs through a DRM domain at the bot- 
tom of the cytopharynx. Cross-reactivity between GM1 and T. cruzi epimastigote DRMs suggests that $T$. cruzi has a not yet identified GM1-homologue glycolipid. A previous study has demonstrated binding of GM1-reactive antibodies to T. cruzi epimastigotes and trypomastigotes (Avila et al. 1998), with identification of gangliosides by HPTLC revealing in trypomastigote extracts the presence of GM1 ( Rf 0.36) with molar ratio of Gal:Glc:GalNac:NeuAc of 1:1:0:1. Our experimental conditions for parasite growth and processing for lipid analysis avoided any contamination with exogenous GM1. Further studies are ongoing in our laboratory with a purified T. cruzi glycolipid fraction, in order to characterize this membrane compound.

The process of transferrin uptake has been extensively analyzed in Trypanosoma brucei (Morgan et al. 2002), but this trypanosome species lacks a cytostome. In T. brucei transferrin is ingested by receptor-mediated endocytosis via clathrin-coated vesicles formed at the flagellar pocket membrane (Coppens et al. 1987, Ligtenberg et al. 1994). As T. cruzi and T. brucei present different life cycles, it is possible that they have developed distinct endocytic mechanisms to exploit they invertebrate and vertebrate hosts.

Endocytosis by lipid rafts represents a clathrin-independent, dynamin-dependent endocytic route (Parton et al. 1994, Nabi \& Le 2003). Raft-related micro-domains are dramatically enriched in cholesterol, sphingolipids, GPIanchored proteins, and lipid-modified signaling molecules (Simons \& Ikonen 1997, Tagawa et al. 2005). Our lipid analysis demonstrated that such micro-domains are present in $T$. cruzi epimastigotes. The presence of lipid raft domains has been previously suggested in trypanosomatids (Nolan et al. 2000, Denny et al. 2001, Denny \& Smith 2004). Studies with Leishmania major and T. brucei have shown lipid rafts as a feature of these parasites (Denny et al. 2001).

The transferrin receptor is a GPI-anchored molecule in T. brucei (Steverding 2000), but there is still no biochemical evidence on transferrin receptors in T. cruzi. A morphological study using freeze-fracture showed absence of integral membrane proteins from the plasma membrane at the cytostome opening (VataruNakamura et al. 2005). Based on these findings, together with our analysis of lipid membrane composition, positive flotillin-1 detection by dot blot in DRM fractions, and mainly the positive co-localization by immunofluorescence of flotillin-1 and TRITC-labeled transferrin at the cytostome/ cytopharynx, it is plausible to suppose that the transferrin receptor in T. cruzi is also GPI-anchored, which would strength our evidence that DRM domains in this parasite can be accepted as lipid raft-like systems.

In T. cruzi epimastigotes, ingested transferrin is transported to and stored at the reservosomes (Soares \& De Souza 1991, De Souza 2002). These organelles are prelysosomal compartments (Soares et al. 1992), and our data suggest that uncoated vesicles budding off from the long cytopharynx are directed to them. Thus, reservosomes may represent late endocytic compartments with DRM domains. If the reservosome is a meeting point of endosomes and DRM domains, we expect that future analyzes of purified reservosome membrane fractions may show the presence of the same markers shown here.

\section{ACKNOWLEDGEMENTS}

To Dr Marcelo Pelajo Machado and Pedro Paulo de A Manso, from Laboratório de Patologia, Instituto Oswaldo Cruz, Rio de Janeiro, for the help with the confocal microscopy.

\section{REFERENCES}

Anderson RGW 1998. The caveolae membrane system. Ann Rev Biochem 67: 199-225.

Ávila JL, Rojas M, Avila A 1998. Increase in asialogangliosideand monosialoganglioside-reactive antibodies in chronic Chagas disease patients. Am J Trop Med Hyg 58: 338-342.

Bendayan M, Nanci A, Kan FW 1987. Effect of tissue processing on colloidal gold cytochemistry. J Histochem Cytochem 35: 983-996.

Bligh EG, Dyer WJ 1959. A rapid method of total lipid extraction and purification. Can J Biochem Physiol 37: 911-917.

Camargo EP 1964. Growth and differentiation in Trypanosoma cruzi. I. Origin of metacyclic trypanosomes in liquid media. Rev Inst Med Trop São Paulo 12: 93-100.

Chung CS, Huang CY, Chang W 2005. Vaccinia virus penetration requires cholesterol and results in specific viral envelope proteins associated with lipid rafts. J Virol 79: 1623-1634.

Coppens I, Opperdoes FR, Courtoy PJ, Baudhuin P 1987. Receptor-mediated-endocytosis in the bloodstream form of Trypanosoma brucei. J Protozool 34: 465-473.

De Souza W 2002. Special organelles of some pathogenic protozoa. Parasitol Res 88: 1013-1025.

Denny PW, Field MC, Smith DF 2001. GPI-anchored proteins and glycoconjugates segregate into lipid rafts in Kinetoplastida. FEBS Lett 491: 148-153.

Denny PW, Smith DF 2004. Rafts and sphingolipid biosynthesis in the kinetoplastid parasitic protozoa. Mol Microbiol 53: 725-733.

El-Sayed NM, Myler PJ, Bartholomeu DC, Nilsson D, Aggarwal G, Tran AN, Ghedin E, Worthey EA, Delcher AL, Blandin G, Westenberger SJ, Caler E, Cerqueira GC, Branche C, Haas B, Anupama A, Arner E, Åslund L, Attipoe P, Bontempi E, Bringaud F, Burton P, Cadag E, Campbell DA, Carrington M, Crabtree J, Darban H, Silveira JF, Jong P, Edwards K, Englund PT, Fazelina G, Feldblyum T, Ferella M, Frasch AC, Gull K, Horn D, Hou L, Huang Y, Kindlund E, Klingbeil M, Kluge S, Koo H, Lacerda D, Levin MJ, Lorenzi H, Louie T, Machado CR, McCulloch R, McKenna A, Mizuno Y, Mottram JC, Nelson S, Ochaya S, Osoegawa K, Pai G, Parsons M, Pentony M, Pettersson U, Pop M, Ramirez JL, Rinta J, Robertson L, Salzberg SL, Sanchez DO, Seyler A, Sharma R, Shetty J, Simpson AJ, Sisk E, Tammi MT, Tarleton R, Teixeira S, Van Aken S, Vogt C, Ward PN, Wickstead B, Wortman J, White O, Fraser CM, Stuart KD, Andersson B 2005. The genome sequence of Trypanosoma cruzi, etiologic agent of Chagas disease. Science 309: 409-415.

Figueiredo RCB, Soares MJ 2000. Low temperature blocks fluidphase pinocytosis and receptor-mediated endocytosis in Trypanosoma cruzi epimastigotes. Parasitol Res 86: 413-418.

Frick M, Bright NA, Riento K, Bray A, Merrified C, Nichols BJ 2007. Coassembly of flotillins induces formation of membrane microdomains, membrane curvature, and vesicle budding. Curr Biol 17: 1151-1156. 
Graf GA, Connell PM, Van der Westhuyzen DR, Smart EJ 1999. The class B, type I scavenger receptor promotes the selective uptake of high-density lipoprotein cholesterol ethers into caveolae. J Biol Chem 274: 12043-12048.

Handcock JF 2006. Lipids rafts: contentious only from simplistic standpoints. Nature 7: 456-462.

Harder T, Scheiffele P, Verkade P, Simons K 1998. Lipid domain structure of the plasma membrane revealed by patching of membrane components. J Cell Biol 141: 929-942.

Harris TJ, Siu CH 2002. Reciprocal raft-receptor interactions and the assembly of adhesion complexes. Bioessays 24: 996-1003.

Ikonen E 2001. Roles of lipid rafts in membrane transport. Curr Opin Cell Biol 13: 470-477.

Kiss AL, Botos E, Turi A, Mullner N 2004. Okadaic acid treatment causes tyrosine phosphorylation of caveolin-2 and induces internalization of caveolae in rat peritoneal macrophages. Micron 35: 707-715.

Ligtenberg MJ, Bitter W, Kieft R, Steverding D, Janssen H, Calafat J, Borst P 1994. Reconstitution of a surface transferrin binding complex in insect form Trypanosoma brucei. EMBO J 13: 2565-2573.

Milder R, Deane MP 1969. The cytostome of Trypanosoma cruzi and T. conorhini. J Protozool 16: 730-737.

Morgan GW, Allen CL, Jeffries TR, Hollinshead M, Field MC 2001. Developmental and morphological regulation of clathrinmediated endocytosis in Trypanosoma brucei. J Cell Sci 114: 2605-2615.

Morgan GW, Hall BS, Denny PW, Carrington M, Field MC 2002. The Kinetoplastida endocytic apparatus. Part I: a dynamic system for nutrition and evasion of host defenses. Trends Parasitol 18: 491-496.

Nabi IR, Le PU 2003. Caveolae/ raft-dependent endocytosis. $J$ Cell Biol 161: 673-677.

Nguyen DH, Hildreth JE 2000. Evidence for budding of human immunodeficiency virus type 1 selectively from glycolipidenriched membrane lipid rafts. J Virol 74: 3264-3272.

Nolan DP, Jackson DG, Biggs MJ., Brabazon ED, Pays A, Van Laethem F, Paturiaux-Hanocq F, Elliott JF, Voorheis HP, E Pays 2000. Characterization of a novel alaninerich protein located in surface microdomains in Trypanosoma brucei.J Biol Chem 275: 4072-4080.

Parton RG, Joggerst B, Simons K 1994. Regulated internalization of caveolae. J Cell Biol 127: 1199-1215.

Porto-Carreiro I, Attias M, Miranda K, De Souza W, Cunha-eSilva N 2000. Trypanosoma cruzi epimastigote endocytic pathway: cargo enters the cytostome and passes through an early endosomal network before storage in reservosomes. Eur J Cell Biol 79: 858-869.

Rietveld A, Simons K 1998. The differential miscibility of lipids as the basis for the formation of functional membrane rafts. Biochim Biophys Acta 1376: 467-479.

Seveau S, Bierne H, Giroux S, Prevost MC, Cossart P 2004. Role of lipid rafts in Ecadherin-and HGF-R/Met-mediated entry of Listeria monocytogenes into host cells. J Cell Biol 166: 743-753.

Simons K, Ikonen E 1997. Functional rafts in cell membranes. Nature 387: 569-572.

Simons K, Toomre D 2000. Lipid rafts and signal transduction. Nature Rev Mol Cell Biol 1: 31-39.

Simons K, Vaz W 2004. Model systems, lipid raft and cell membrane. Ann Rev Biophys Biomol Struct 33: 269-295.

Slot JW, Geuze HJ 1985. Anew method of preparing gold probes for multiple-labeling cytochemistry. Eur J Cell Biol 38: 87-93.

Soares MJ, De Souza W 1988. Cytoplasmic organelles of trypanosomatids: a cytochemical and stereological study. $J$ Submicrosc Cytol Pathol 20: 349-361.

Soares MJ, De Souza W 1991. Endocytosis of gold-labeled proteins and LDL by Trypanosoma cruzi. Parasitol Res 77: 461-468.

Soares MJ, Souto-Padrón T, De Souza W 1992. Identification of a large pre-lysosomal compartment in the pathogenic protozoon Trypanosoma cruzi. J Cell Sci 102: 157-167.

Steverding D 2000. The transferrin receptor of Trypanosoma brucei. Parasitol Int 48: 191-198.

Tagawa A, Mezzacasa A, Hayer A, Longatti A, Pelkmans L, Helenius A 2005. Assembly and trafficking of caveolar domains in the cell: caveolae as stable, cargo-triggered, vesicular transporters. J Cell Biol 170: 769-779.

VataruNakamura C, Ueda-Nakamura T, De Souza W 2005. Visualization of the cytostome in Trypanosoma cruzi by high resolution field emission scanning electron microscopy using secondary and backscattered electron imaging. FEMS Microbiol Lett 242: 227-230.

Vogel WC, Doizaki WM, Zieve L 1962. Rapid thin-layer chromatographic separation of phospholipids and neutral lipids of serum. J Lipid Res 3: 138-140.

Yavin E, Zutra A 1977. Separation and analysis of ${ }^{32} \mathrm{P}-$ labeled phospholipids by a simple and rapid thin-layer chromatographic procedure and its application to cultured neuroblastoma cells. Anal Biochem 80: 430-437. 